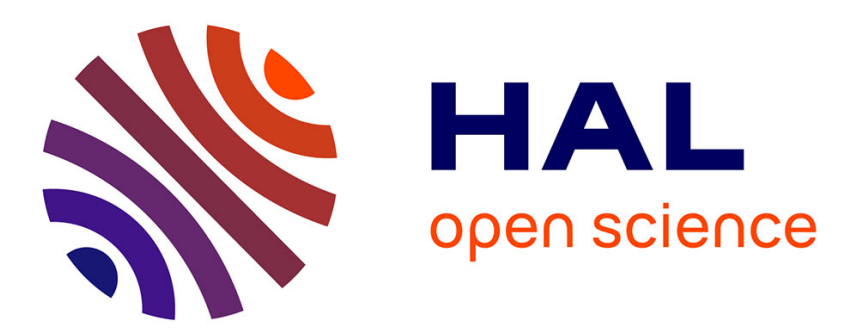

\title{
Recurrence phase shift in Fermi-Pasta-Ulam nonlinear dynamics
}

\author{
N. Devine, A. Ankiewicz, G. Genty, J.M. Dudley, N. Akhmediev
}

\section{To cite this version:}

N. Devine, A. Ankiewicz, G. Genty, J.M. Dudley, N. Akhmediev. Recurrence phase shift in Fermi-Pasta-Ulam nonlinear dynamics. Physics Letters A, 2011, 375 (46), pp.4158-4161. 10.1016/j.physleta.2011.10.006 . hal-00655031

\section{HAL Id: hal-00655031 \\ https://hal.science/hal-00655031}

Submitted on 29 Apr 2021

HAL is a multi-disciplinary open access archive for the deposit and dissemination of scientific research documents, whether they are published or not. The documents may come from teaching and research institutions in France or abroad, or from public or private research centers.
L'archive ouverte pluridisciplinaire HAL, est destinée au dépôt et à la diffusion de documents scientifiques de niveau recherche, publiés ou non, émanant des établissements d'enseignement et de recherche français ou étrangers, des laboratoires publics ou privés. 


\title{
Recurrence phase shift in Fermi-Pasta-Ulam nonlinear dynamics
}

\author{
N. Devine ${ }^{\mathrm{a}, *}$, A. Ankiewicz ${ }^{\mathrm{a}}$, G. Genty ${ }^{\mathrm{b}}$, J.M. Dudley ${ }^{\mathrm{c}}$, N. Akhmediev ${ }^{\mathrm{a}}$ \\ a Optical Sciences Group, Research School of Physics and Engineering, The Australian National University, Canberra ACT 0200, Australia \\ b Tampere University of Technology, Optics Laboratory, FI-33101 Tampere, Finland \\ c Institut FEMTO-ST UMR 6174 CNRS/Université de Franche-Comté, Besançon, France
}

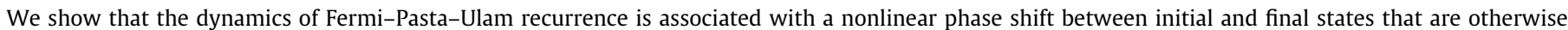

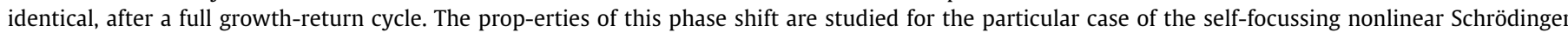

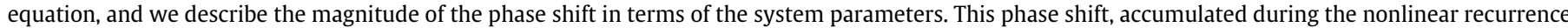

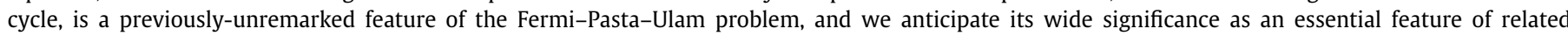
dynamics in other systems.

Many nonlinear systems in physics display the universal dynamical feature of Fermi-Pasta-Ulam (FPU) recurrence [1-11]. The study of FPU recurrence dates back to 1955 when Fermi, Pasta and Ulam numerically simulated the evolution dynamics of a nonlinear oscillator chain, subject to initial excitation in just a single normal mode. Although the energy would remain entirely within this initial excited mode in a linear system, the presence of nonlinear coupling led FPU to expect that energy would be transferred from the initial mode to the other modes of the system in a process of energy equipartition. However, what FPU actually observed was a remarkable phenomenon where, although energy was at first transferred from the initially-excited mode into the higher-order modes of the system, subsequent evolution resulted in the transfer of the energy back to the initial state, with all other modes returning to the ground state with zero excitation. Since its initial and unexpected discovery, the effect of "FPU recurrence" has become a defining feature of nonlinear dynamical systems, and has been related to the mathematics of integrable systems and nonlinear localization and soliton physics [12].

Surprisingly, however, one aspect of FPU recurrence has not been completely addressed in previous studies. In particular, because the state of any oscillator is defined not only by its amplitude, but also by its phase, the question naturally arises as to whether the states of a system undergoing FPU recurrence have the same phase at the beginning and at the end of the process. Certainly, studies of phase-space trajectories in general have provided much insight into the dynamics and chaos of nonlinear systems, but the specific question relating to a global system phase

\footnotetext{
* Corresponding author. Tel.: +61 26125 9984; fax: +61261259476.

E-mail address: nnd124@rsphysse.anu.edu.au (N. Devine).
}

shift in FPU recurrence has not been explicitly examined. This is somewhat surprising, because it is well known, from studies of linear systems, that phase evolution can be intimately linked to the fundamental symmetries of a system under study $[13,14]$.

Our results in this Letter reveal the corresponding importance of the phase shift between initial and final states as a defining parameter of FPU recurrence in the context of nonlinear dynamics. Notably, although a superficial examination of the system state (e.g. in terms of energy) will suggest that initial and final states are identical, we show that the nonlinear evolution linking these two points leaves its trace embedded in the form of a constant phase shift. This phase shift introduces an important physical distinguishability between two states that may otherwise be considered to be identical in a system.

Although FPU dynamics can be observed in many different systems, we introduce the notion of a recurrence phase shift in the context of the self-focussing nonlinear Schrödinger equation (NLSE). We hope that the clear results obtained for this particular case will motivate further studies extending these ideas into other systems. The NLSE, in dimensionless form, is given by:

$i \frac{\partial \psi}{\partial t}+\frac{1}{2} \frac{\partial^{2} \psi}{\partial x^{2}}+|\psi|^{2} \psi=0$

The dynamics of interest here relates to modulation instability, the growth and decay of an initial small amplitude modulation on a plane wave solution to Eq. (1) [15-20]. The dynamics of modulation instability has recently been shown to be very well described by an exact family of solutions to Eq. (1) that solve the FPU problem exactly [21-23]. It is these analytic results [24,25] that we apply here to study the nonlinear phase shift accumulation in detail. These solutions, known as Akhmediev breathers (AB) [26-30] are 

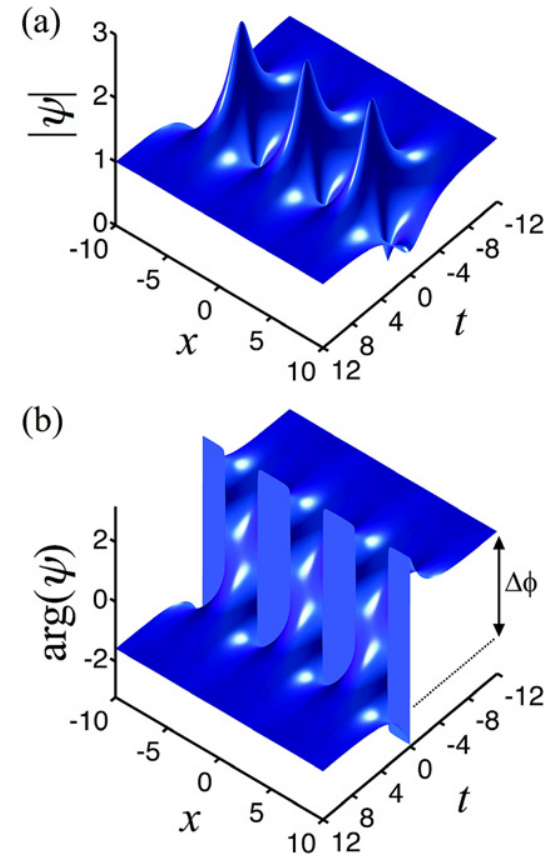

Fig. 1. (a) Amplitude of the $\mathrm{AB}$ solution defined by Eq. (2) with $a=0.25$. The evolution starts with a constant background, $q=1$, which is slightly modulated. The modulation grows to reach its maximum at $t=0$, and finally the solution returns to the original background, $q=1$. (b) Phase evolution $\arg [\psi(x, t)]$ for the same solution. The figure illustrates how the amplitude returns to its initial plane wave state after the FPU cycle, even though the field develops a phase shift of $\Delta \phi$ relative to the initial value during nonlinear propagation.

given by

$\psi(x, t)=\left[1-\frac{2(1-2 a) \cosh (\delta t)+i \delta \sinh (\delta t)}{\cosh (\delta t)-\sqrt{2 a} \cos (\kappa x)}\right] e^{i t}$.

The free parameter $a$ varies in the interval from 0 to $1 / 2$, and determines the spatial frequency of initial modulation $\kappa=$ $2 \sqrt{(1-2 a)}$ (so that $0<\kappa<2)$ and the initial instability growth rate $\delta=\sqrt{8 a(1-2 a)}=\kappa \sqrt{1-\kappa^{2} / 4}$. An additional scaling parameter $q$ can be added to any solution of the NLSE [31]: $\psi^{\prime}(x, t)=$ $q \psi\left(q x, q^{2} t\right)$. This transformation renormalizes the background to any desired value, $q$, and simultaneously rescales the two independent variables, $x$ and $t$. However, the rescaling does not influence the phases and we will not explicitly use it in what follows.

The solution of Eq. (2) is shown in Fig. 1, where we plot (a) the amplitude and (b) the phase of the solution. Note that the phase factor $\exp (i t)$ associated with purely linear propagation is removed in order to highlight the specific contribution of the nonlinear evolution. The dynamics here begins from a constant background, infinitesimally modulated at frequency $\kappa$ along $x$. The modulation grows exponentially, with the creation of new spectral components at harmonics of $\kappa$. In the frequency domain, the exponential amplification is associated with energy transfer from the central (pump) mode to higher harmonics, but after the growth to the point of peak amplitude is completed, the process is reversed and the system returns to its initial state.

The amplitude dynamics of this process has been the subject of many studies (see Ref. [22] and references therein), but what appears to have gone unremarked is the presence of the nonlinear phase shift between the initial and final states. This is indicated explicitly as $\Delta \phi$ in Fig. 1(b). This figure clearly shows how the nonlinear propagation fundamentally modifies the physical state of the system, even though the plane wave amplitude [in Fig. 1(b)] has been recovered during the return cycle.

The corresponding dynamics for the frequency domain spectral modes can also be calculated exactly:

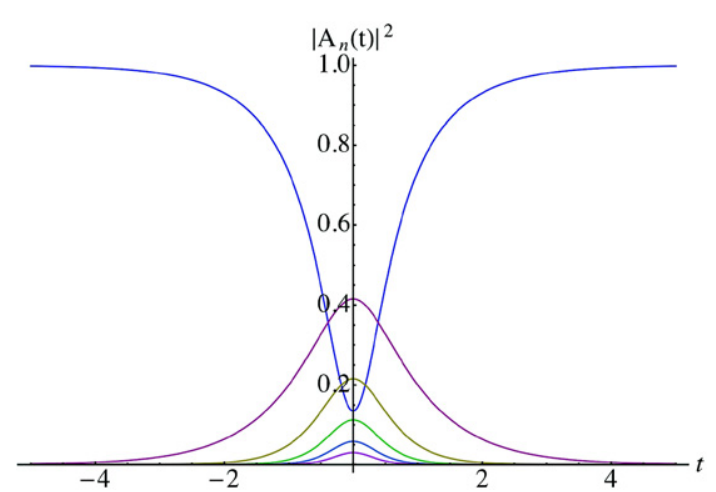

Fig. 2. (Color online.) Evolution of the energy spectra of the carrier wave (upper curve) and five nearest sidebands $n= \pm 1, \pm 2, \pm 3, \pm 4, \pm 5$ (counting down). Parameter $a$ is 0.45 . Note that, for the sidebands, we plot the sum of both $\pm n$ harmonics for clarity.

$$
\begin{aligned}
A_{0}(t)= & 1-\frac{i \delta \sinh (\delta t)+\left(\kappa^{2} / 2\right) \cosh (\delta t)}{\sqrt{\cosh ^{2}(\delta t)-2 a}}, \\
A_{n}(t)= & \frac{i \delta \sinh \delta t+\left(\kappa^{2} / 2\right) \cosh (\delta t)}{\sqrt{\cosh ^{2}(\delta t)-2 a}} \\
& \times\left[\frac{\cosh (\delta t)-\sqrt{\cosh ^{2}(\delta t)-2 a}}{\sqrt{2 a}}\right]^{|n|} .
\end{aligned}
$$

Here $A_{0}(t)$ describes the $t$-evolution of the (initial) pump mode and the $A_{n}(t)$ correspond to the harmonics $n= \pm 1, \pm 2, \ldots$ Parameters $a, \kappa$ and $\delta$ are as above. As the total energy is conserved, the spectral components satisfy the equation: $\left|A_{0}(t)\right|^{2}+$ $2 \sum_{n=1}^{\infty}\left|A_{n}(t)\right|^{2}=1$.

The time dependence for each of these components, up to $n=$ \pm 5 , is shown in Fig. 2. We see clearly how the central spectral component contains the total energy at $t \rightarrow-\infty$, and how this is redistributed during the evolution to harmonic sidebands before returning to the central mode at the end of the process, $t \rightarrow \infty$. The phase shift experienced by the central mode can be readily calculated from Eq. (3) as:

$\Delta \phi=2 \arccos \left(\frac{\kappa^{2}}{2}-1=2 \arccos (1-4 a)\right.$.

This is easy to see if we consider the position of points defined by Eq. (3) on the complex plane at $t \rightarrow-\infty$ and $t \rightarrow \infty$. These are located on the unit circle in the complex plane with the real part equal to $\left(1-\kappa^{2} / 2\right)$. This phase shift is, of course, identical to the phase shift experienced by the corresponding plane wave in the time domain, which can be determined from Eq. (2)

The phase shift given by Eq. (5) is an intrinsic consequence of the FPU recurrence dynamics. We can make the following observations. Firstly, the magnitude of $\Delta \phi$ depends on the physical modulation frequency of the initial plane wave $\kappa$, as shown in Fig. 3. For low modulation frequency, $\kappa=0(a=1 / 2)$, where the solution corresponds to the Peregrine soliton [22,32], $\Delta \phi$ attains a maximal value of $2 \pi$. For a high modulation frequency at highest edge of the instability band, $\kappa=2(a=0)$, the phase shift tends to zero.

The exact results for the frequency domain dynamics in Eqs. (3) and (4) allow the dynamical variation of the phase shift across the spectral components to be readily calculated and explicitly plotted as a function of the evolution variable $t$ for all modulation parameters. This is shown in Fig. 4 which plots the evolution with $t$ of the phase of both the pump $A_{0}$ and the higher-order sidebands $A_{n}$ (all of which have the same phase) for all modulation parameters $a$ spanning the range 0 to $1 / 2$. These graphs complement the 

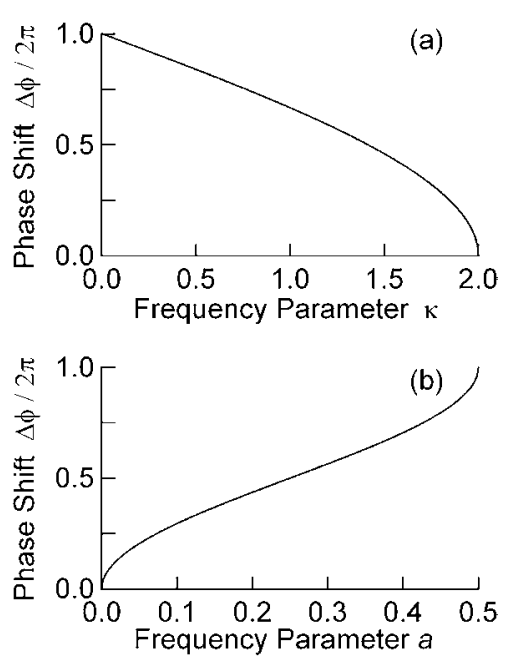

Fig. 3. The figure (a) show how the phase shift related to FPU recurrence depends on the modulation frequency parameter $\kappa$ and spans the full range from 0 to $2 \pi$. For convenience, (b) plots the equivalent curve against the parameter $a=\left(1-\kappa^{2} / 4\right) / 2$.
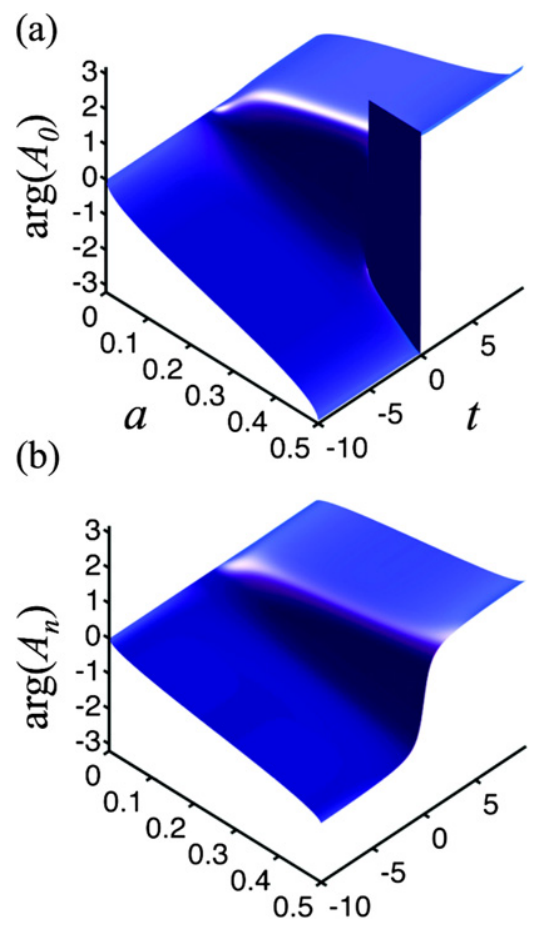

Fig. 4. Phase shift of pump $A_{0}$ and sideband components $A_{n}$ calculated as a function of $t$ for different modulation parameters $a$.

time-domain representation as shown in Fig. 1 in revealing the rich behavior present in the phase dynamics.

Another way to visualize the recurrence and the corresponding phase shift is to view the complex plane of solutions as shown in Fig. 5, defined by Eq. (2) with $x=0$. The phase shift gained by the central frequency component is the same as the phase shift between the plane wave solutions at $t \rightarrow-\infty$ and $t \rightarrow \infty$. For any $x$, trajectories in the complex plane connect two points located on the unit circle, as shown in Fig. 5, clearly demonstrating that there is a phase shift after recurrence, even though the amplitudes (the points on the circle) remain unchanged. In general, a solution at the point $\exp (i \Delta \phi / 2)$ is transformed to the point $\exp (-i \Delta \phi / 2)$ on the circle; this is also a constant background solution, but with shifted phase. In the case with the maximum growth rate, when $a=0.25$, the shift is $\pi$. Thus, after the breather has developed,

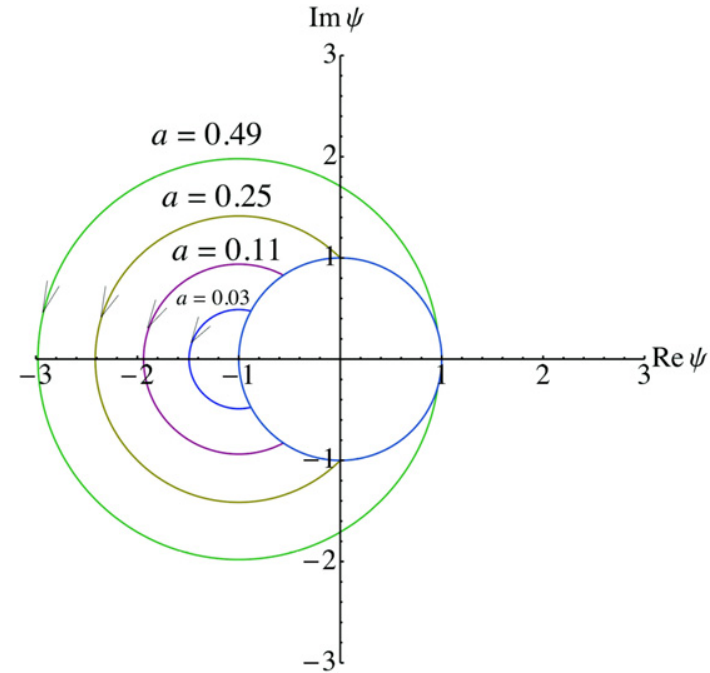

Fig. 5. Trajectories in the complex plane demonstrating FPU recurrence and the phase shift related to it. Four trajectories, defined by Eq. (2), are plotted for the parameter $a=0.03 ; 0.11 ; 0.25 ; 0.49$. A unit circle with its centre at the origin defines the points of initial and final complex amplitudes of the plane wave. Start and end points on the unit circle do not depend on $x$, while the actual trajectories do. Here, the variable $x=0$.
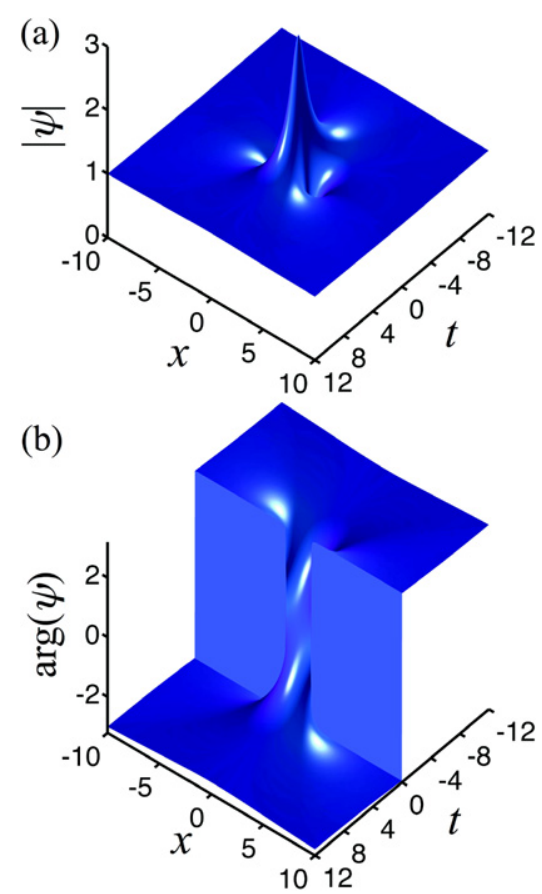

Fig. 6. Peregrine soliton, defined by Eq. (2), with $a \rightarrow 0.5$, showing the single spatiotemporal peak observed in this limit. Phase diagram $\arg [\psi(t)]$ for the same solution The nonlinear phase shift $\Delta \phi$ in this case is $2 \pi$.

the plane wave has gained a phase shift of $\Delta \phi$ as a result of the nonlinear energy exchange of the evolving field with the background.

A special case of Eq. (2) when $a \rightarrow 0.5$ (i.e. when the period along the $x$-axis $\rightarrow \infty$ is the Peregrine soliton shown in Fig. 6(a) and given by:

$\psi_{1}=\left(1-4 \frac{1+2 i t}{1+4 x^{2}+4 t^{2}} \quad e^{i t}\right.$.

This solution is strongly localized, both in space and time, as shown in Fig. 6(a), and the phase shift relative to the background for this solution is $2 \pi$, as shown in Fig. 6(b). However, 
it is important to note that, although the final phase returns to the original phase of the background, the evolution is associated with the wave field changing phase through all intermediate values from 0 to $2 \pi$. These changes dramatically influence the wave carrier in the immediate proximity to the solution, which is particularly relevant in this case, as the Peregrine soliton is often considered as a prototype of rogue waves in the ocean where rapid phase changes of the carrier would clearly have dramatic consequences [30].

The principal result of this Letter has been to identify a nonzero phase shift which modifies the state of a plane wave field before and after an FPU growth-return cycle. The accumulated phase shift varies from 0 to $2 \pi$, depending on the position of the initial perturbation within the instability band (as shown in Fig. 3). Although we have considered the specific case of FPU dynamics in the NLSE, we expect that the development of a phase shift across an FPU recurrence cycle will be a generic effect for a large class of nonlinear systems.

We can also speculate upon the consequences of our results when interpreting the observed behavior of other systems which show rapid growth and decay of some initial perturbation. Such events occur widely in many different areas of science, and include such processes as periodic flooding, financial cycles of boom and bust, and also social instability phenomena like demonstrations and revolutions. Although each of these systems requires specific modeling scenarios to be analyzed in detail, the significance of our results here is that they suggest that, for a system that undergoes some type of rapid growth-decay evolution, even though it may appear to have returned to a state that is identical with its initial one, a residual signature of its nonlinear evolution remains in its nonlinear phase shift. When such systems subsequently evolve in the presence of coupling and synchronization with other processes, it is clear that a nonlinearly acquired phase shift may have a dramatic influence. Of course, we stress that whilst a single phase parameter is unlikely to capture the full complexity of all processes of this sort, future studies of nonlinear phase developed during FPU recurrence may well have significant impacts in many different areas of physics.

\section{Acknowledgements}

The authors acknowledge the support of the A.R.C. (Discovery Project DP110102068). One of the authors - N.A. - is a recipient of the Alexander von Humboldt Award (Germany). J.M.D. acknowledges support from the project IMFINI ANR-09-BLAN-0065.

\section{References}

[1] E. Fermi, J. Pasta, S. Ulam, Los Alamos report LA-1940, 1955; reprinted in: E. Segré (Ed.), E. Fermi, Collected Papers, vol. II, Univ. Chicago Press, 1965, p. 978.

[2] R.L. Bivins, N. Metropolis, J. Pasta, J. Comput. Phys. 12 (1973) 65

[3] D.S. Sholl, B.I. Henry, Phys. Rev. A 44 (1991) 6363.

[4] J. Ford, Phys. Rep. 213 (1992) 271.

[5] K. Yoshimura, Phys. Rev. E 62 (2000) 6447.

[6] N. Akhmediev, Nature 413 (2001) 267.

[7] T. Dauxois, M. Peyrard, S. Ruffo, Eur. J. Phys. 26 (2005) S3.

[8] S. Flach, M.V. Ivanchenko, O.I. Kanakov, Phys. Rev. Lett. 95 (2005) 064102

[9] M. Wu, C.E. Patton, Phys. Rev. Lett. 98 (2007) 047202.

[10] V.P. Ruban, arXiv:1012.3246v2 [physics.flu-dyn], 15 February 2011.

[11] V.P. Ruban, arXiv:1104.0853v1 [physics.flu-dyn], 5 April 2011.

[12] N.J. Zabusky, M.D. Kruskal, Phys. Rev. Lett. 15 (1965) 240.

[13] M. Berry, Proc. R. Soc. Lond. A 392 (1984) 45.

[14] J. Anandan, J. Christian, K. Wanelik, Am. J. Phys. 65 (1997) 180.

[15] V.I. Bespalov, V.I. Talanov, Zh. Eksp. Teor. Fiz. Pis'ma Red. 3 (1966) 471; English translation: JETP Lett. 3 (1966) 307.

[16] T.B. Benjamin, J.E. Feir, Theory J. Fluid Mech. 27 (1967) 417.

[17] H.C. Yuen, J. Warren, W.E. Ferguson, Phys. Fluids 21 (1978) 1275

[18] E. Infeld, Phys. Rev. Lett. 47 (1981) 717.

[19] G. Van Simaeys, Ph. Emplit, M. Haelterman, J. Opt. Soc. Am. B 19 (2002) 477.

[20] J. Beeckman, X. Hutsebaut, M. Haelterman, K. Neyts, Opt. Express 15 (2007) 11185.

[21] J.M. Dudley, G. Genty, F. Dias, B. Kibler, N. Akhmediev, Opt. Express 17 (2010) 21497.

[22] B. Kibler, J. Fatome, C. Finot, G. Millot, F. Dias, G. Genty, N. Akhmediev, J.M. Dudley, Nature Phys. 6 (2010) 790.

[23] K. Hammani, B. Kibler, C. Finot, P. Morin, J. Fatome, J.M. Dudley, G. Millot, Opt. Lett. 36 (2011) 112.

[24] N. Akhmediev, V.I. Korneev, Theor. Math. Phys. (USSR) 69 (2) (1986) 1089; Translation from Russian: Teoret. Mat. Fiz. 69 (1986) 189.

[25] N. Akhmediev, V.M. Eleonckii, N.E. Kulagin, Theor. Math. Phys. (USSR) 72 (1987) 809; Translation from Russian: Teoret. Mat. Fiz. 72 (1987) 183.

[26] K.B. Dysthe, K. Trulsen, Phys. Scr. T 82 (1999) 48.

[27] I. Ten, H. Tomita, Reports of RIAM Symposium No. 17SP1-2, Proceedings of a symposium held at Chikushi Campus, Kyushu University, Kasuga, Fukuoka, Japan, 10-11 March 2006

[28] D. Clamond, M. Francius, J. Grue, C. Kharif, Eur. J. Mech. B - Fluids 25 (2006) 536.

[29] V.V. Voronovich, V.I. Shrira, G. Thomas, J. Fluid Mech. 604 (2008) 263.

[30] V.I. Shrira, V.V. Geogjaev, J. Eng. Math. 67 (2010) 11.

[31] N. Akhmediev, A. Ankiewicz, Solitons, Nonlinear Pulses and Beams, Chapman and Hall, London, 1997.

[32] D.H. Peregrine, J. Australian Math. Soc. Ser. B 25 (1983) 16. 